\title{
SPETTROSCOPIA E IMAGING IN OTTICA DIFFUSA: FOCUS SUL TUMORE DELLA MAMMELLA
}

\author{
PAOLA TARONI (*) \\ Nota presentata dal m.e. Antonio Pedotti \\ (Adunanza del 23 maggio 2019)
}

\begin{abstract}
SuNTO. - Attraverso la misura delle proprietà ottiche (assorbimento e diffusione), la spettroscopia ottica diffusa consente la stima non invasiva della composizione dei tessuti biologici (contenuto di acqua, lipidi e collagene) e dei parametri funzionali del sangue e fornisce informazioni sulla struttura microscopica del tessuto. Può quindi essere efficacemente utilizzata in vivo come strumento diagnostico assolutamente non invasivo. Il Dipartimento di Fisica del Politecnico di Milano ha progettato e realizzato un mammografo ottico che sfrutta proprio l'ottica diffusa, operando con luce impulsata a 7 lunghezze d'onda nel rosso e vicino infrarosso $(635-1060 \mathrm{~nm})$. Lo strumento è stato utilizzato in uno studio clinico condotto su 200 soggetti, in collaborazione con l'Istituto Europeo di Oncologia: la composizione tissutale derivata otticamente e in particolare il contenuto di collagene nei tessuti si sono rivelati efficaci sia per discriminare tra lesioni mammarie maligne e benigne, che per stimare il rischio di tumore della mammella legato alla densità del tessuto mammario, che è riconosciuta tra i più importanti fattori indipendenti di rischio. Anche sulla base di questi risultati, "SOLUS - Smart optical and ultrasound diagnostics of breast cancer", progetto europeo del Programma Quadro H2020, sta ora lavorando per migliorare la specificità della diagnosi non invasiva del tumore della mammella combinando ottica diffusa con imaging a ultrasuoni.
\end{abstract}

$* * *$

ABSTRACT. - Through the measurement of the optical properties (absorption and scattering), diffuse optical spectroscopy allows one to estimate non-invasively the composition of biological tissues (water, lipid and collagen content) and functional blood parameters. Further, it provides information on the microscopic tissue structure. It can the-

(") Dipartimento di Fisica, Politecnico di Milano, Italy.

E-mail: paola.taroni@polimi.it 
refore be effectively used in vivo as an absolutely non-invasive diagnostic tool. The Department of Physics of the Politecnico di Milano has designed and built an optical mammograph that exploits diffused optics, operating with pulsed light at 7 wavelengths in the red and near infrared spectral range $(635-1060 \mathrm{~nm})$. The instrument was used in a clinical study on 200 subjects, in collaboration with the European Institute of Oncology: optically derived tissue composition and in particular collagen content in tissues proved to be effective both to discriminate between malignant and benign breast lesions, and to estimate the risk of breast cancer related to the density of breast tissue, which is recognized among the most important independent risk factors. Partly based on those results, "SOLUS - Smart optical and ultrasound diagnostics of breast cancer", a European project in the H2020 Framework Program, is now working to improve the specificity of non-invasive breast cancer diagnosis by combining diffuse optics with ultrasound imaging.

\section{L'OTTICA DIFFUSA E I TESSUTI BIOLOGICI}

Quando la luce viene iniettata in un tessuto biologico, solo una piccola frazione viene riflessa direttamente dalla superficie. La maggior parte della luce entra nel mezzo e viene assorbita o diffusa al suo interno.

Sia l'assorbimento che la diffusione (scattering) sono fortemente dipendenti dalla lunghezza d'onda: nel visibile si verificano con probabilità comparabile, mentre a lunghezze d'onda maggiori (rosso e vicino infrarosso, near infrared - NIR) gli eventi di scattering diventano dominanti, ma entrambi i processi sono molto meno probabili, consentendo un penetrazione della luce molto maggiore. Di conseguenza, per avere accesso a strati di tessuto più profondi per scopi diagnostici, vengono solitamente scelte lunghezze d'onda comprese tra 600 e $1100 \mathrm{~nm}$.

Attraverso un modello di propagazione della luce nel tessuto si ottiene poi una correlazione tra il modo in cui la luce viene assorbita e diffusa durante la propagazione attraverso un mezzo e le proprietà ottiche del mezzo stesso (essenzialmente la probabilità di assorbimento o scattering per unità di lunghezza, cioè i coefficienti di assorbimento e di scattering $m_{a}$ e $m_{s}$, rispettivamente). In pratica, i fotoni sopravvissuti alla riflessione o trasmissione diffusa (a seconda della geometria di misura) vengono rilevati e forniscono informazioni sul mezzo attraversato.

Il trasporto della luce attraverso mezzi altamente diffusivi può in linea di principio essere descritto utilizzando la teoria analitica basata direttamente sulle equazioni di Maxwell e tenendo conto della natura 
ondulatoria della luce. Tuttavia, a causa della complessa struttura del tessuto, l'applicazione della teoria elettromagnetica è estremamente difficile e difficilmente utilizzabile nella pratica. In alternativa, può essere considerata la teoria del trasporto radiativo. Basandosi sulla natura particellare della luce, essa descrive il trasporto di fotoni nel tessuto come la propagazione di un flusso di particelle, come avviene, ad esempio, per i fasci di neutroni. La teoria del trasporto è matematicamente meno rigorosa della teoria elettromagnetica e ulteriori approssimazioni (che portano alla cosiddetta "teoria della diffusione") devono essere introdotte per ottenere una soluzione analitica del problema: gli eventi di scattering devono essere isotropi e fortemente dominanti sugli eventi di assorbimento e diversi eventi di scattering devono verificarsi nel mezzo prima che la descrizione fornita diventi sufficientemente accurata [1]. In particolare, per far coincidere l'ipotesi di scattering isotropo con l'evidenza sperimentale della forte anisotropia tipicamente osservata in un tessuto biologico, viene introdotto il coefficiente di scattering ridotto $\mu_{s}^{\prime}$, con $\mu_{s}^{\prime}=\mu_{s}(1-g)$, dove g è il fattore di anisotropia, cioè il coseno medio dell'angolo di diffusione. Nonostante i suoi limiti, l'approssimazione della diffusione alla teoria del trasporto radiativo si è dimostrata ampiamente utile per descrivere il trasporto della luce nei tessuti biologici. In realtà, soluzioni esatte dell'equazione del trasporto radiativo sono state recentemente introdotte per geometrie specifiche e sono disponibili altri modelli teorici (ad esempio, onde di densità di fotoni [2]. Tuttavia, l'approssimazione della diffusione è ancora l'approccio teorico più utilizzato in campo biomedico, perché le sue ipotesi principali sono soddisfatte nel vicino infrarosso e fornisce una semplice soluzione analitica al problema della propagazione dei fotoni in mezzi altamente diffusivi, come i tessuti biologici.

Una delle applicazioni diagnostiche dell'ottica diffusa che negli anni ha generato maggiore interesse è la diagnosi del tumore della mammella (mammografia ottica). In breve, l'idea è simile a quella alla base della convenzionale mammografia a raggi X. Nel caso ottico, radiazione di bassa potenza, nel vicino infrarosso, viene iniettata nella mammella e la luce rivelata dopo essersi propagata attraverso il tessuto mammario porta informazioni sulle sue proprietà ottiche e su eventuali disomogeneità ottiche, rivelando la presenza e la natura di situazioni patologiche.

Affinché una tale tecnica possa essere utile dal punto di vista diagnostico, è innanzitutto essenziale conoscere le proprietà ottiche di 
un tessuto sano e come queste siano influenzate dall'insorgere di una condizione patologica. Tuttavia, il tessuto mammario stesso non è un mezzo otticamente omogeneo e mostra anzi una complessa struttura eterogenea, come prevedibile in base alla sua morfologia. Analogamente a quanto normalmente osservato per altri parametri biologici, le proprietà ottiche del tessuto mammario possono variare sensibilmente da soggetto a soggetto, anche se appartengono alla stessa categoria per quanto riguarda i parametri demografici (ad esempio, hanno la stessa età o lo stesso indice di massa corporea). Inoltre, i cambiamenti fisiologici, come quelli che si verificano durante il ciclo mestruale, la gravidanza o l'allattamento, possono influenzare fortemente le proprietà ottiche del tessuto mammario. Questo, insieme alla variabilità biologica inter- e intra-soggetto sopra menzionata, rende l'indagine del tessuto biologico da un punto di vista ottico piuttosto complessa.

\section{TECNICHE DI MISURA}

Per quanto riguarda le tecniche sperimentali, possono essere previsti tre diversi approcci per stimare le proprietà ottiche di un mezzo altamente diffusivo come un tessuto biologico. La configurazione più semplice implica l'uso di luce in stato stazionario (continuous wave - CW) per eseguire misure dell'attenuazione della luce causata dalla propagazione attraverso il mezzo studiato. L'attenuazione generalmente dipende sia dalle proprietà di assorbimento che di scattering. Perciò una singola misura di attenuazione non consente di separare i contributi dei due fenomeni. Per caratterizzare un mezzo, in generale vengono eseguite più misure a diverse distanze tra sorgente e rivelatore.

Le tecniche che lavorano nel dominio della frequenza misurano l'intensità di luce modulata sinusoidalmente ad almeno una frequenza, tipicamente di poche centinaia di MHz. La propagazione in un mezzo biologico provoca attenuazione (demodulazione) e ritardo di fase della luce riemessa rispetto a quella iniettata. La modulazione e lo sfasamento del segnale rivelato sono legati ai coefficienti ottici del mezzo ed i loro valori, interpretati utilizzando un modello come la teoria della diffusione, consentono la stima sia di $\mu_{a}$ che di $\mu_{s}^{\prime}$.

Infine, l'approccio che opera nel dominio del tempo implica 
l'uso di brevi impulsi luminosi (con durata tipica dell'ordine di decine di picosecondi). Quando iniettato in un mezzo diffusivo, un impulso luminoso è fortemente attenuato (fino a 5-7 ordini di grandezza nelle applicazioni pratiche), ma anche allargato (fino ad alcuni nanosecondi) e ritardato, principalmente a causa delle proprietà di diffusione del mezzo attraversato. Più in dettaglio, ci aspettiamo che gli eventi di scattering rendano le traiettorie dei fotoni diverse tra loro e molto più lunghe della distanza tra i punti di iniezione e di raccolta, ritardando così in modo significativo i fotoni rivelati e ampliando l'intervallo di tempo nel quale vengono rivelati rispetto alla durata dell'impulso incidente. Gli effetti dei processi di assorbimento sono più evidenti sulla coda dell'impulso, dove si rilevano fotoni che sono rimasti più a lungo nel mezzo, sperimentando così una maggiore probabilità di essere assorbiti. Pertanto l'assorbimento, rimuovendo fotoni a tempi lunghi, aumenta la pendenza negativa della coda dell'impulso. Ritardo e cambiamenti di forma dell'impulso rivelato possono essere correlati ai due coefficienti ottici e consentono la loro stima da una singola misura in riflessione o trasmissione. In questo modo non è necessario utilizzare informazioni derivate dall'ampiezza dell'impulso e si evitano così tutti i problemi pratici legati alla stima assoluta dell'intensità luminosa (problemi che, al contrario, possono influenzare in modo significativo le misurazioni $\mathrm{CW}$ ).

La dualità degli studi risolti in frequenza e in tempo è evidente, se si considera che la trasformata di Fourier consente di passare da un dominio all'altro. Tuttavia, si dovrebbe tener conto che in linea di principio tutte le frequenze sono contenute in una misura risolta nel tempo e anche in pratica la gamma di frequenze che corrispondono ad una misura nel dominio del tempo è molto ampia e limitata solo dalla risoluzione temporale dell'apparato sperimentale. Pertanto, una singola misura risolta nel tempo garantirà un contenuto informativo più elevato rispetto a un'acquisizione risolta in frequenza eseguita a una singola frequenza o a un limitato numero di frequenze. Per cercare di compensare questo limite, i sistemi più sofisticati che lavorano in frequenza utilizzano un sintetizzatore per eseguire la scansione di un'ampia gamma di frequenze.

In passato la necessità di strumentazione più complessa e costosa ha limitato la diffusione delle tecniche che operano nel dominio del tempo. Tuttavia, recentemente il rapido sviluppo tecnologico in campo optoelettronico ha fortemente promosso l'utilizzo di sistemi risolti nel 
tempo, che stanno diventando una scelta comune ed efficace non solo per l'uso di laboratorio, ma anche in applicazioni cliniche.

\section{Il mammografo OtTiCo Del Politecnico di Milano}

\subsection{La configurazione dello strumento}

Lo strumento è stato progettato per acquisire immagini in proiezione con la mammella in compressione, nella stessa configurazione della convenzionale mammografia a raggi X (Fig. 1). Sette laser a diodi impulsati vengono utilizzati come sorgenti luminose che emettono a 635, 680 e $785 \mathrm{~nm}$ (visibile, VIS) e a 905, 930, 975 e $1060 \mathrm{~nm}$ (vicino infrarosso, NIR), con potenza media di $\sim 1-5 \mathrm{~mW}$, durata dell'impulso di $\sim 150-400$ ps e frequenza di ripetizione di $20 \mathrm{MHz}$. La mammella è compresso tra lastre di vetro temperato parallele, con un grado di compressione molto più modesto che nel caso della mammografia $\mathrm{X}$, dato che la lunghezza della misura è maggiore. Nella versione dello strumento utilizzata per lo studio descritto nel seguito, la luce trasmessa attraverso la mammella veniva raccolta da un fascio di fibre, la cui estremità distale era biforcata e i due bracci guidavano i fotoni a un tubo fotomoltiplicatore (PMT) per la rivelazione delle lunghezze d'onda VIS (R5900U-01-L16, Hamamatsu, Giappone) e a un PMT per le lunghezze d'onda NIR (sensibile fino a $1100 \mathrm{~nm}, \mathrm{H} 7422 \mathrm{P}-60$, Hamamatsu, Giappone), rispettivamente. Due schede (SPC130, Becker \& Hickl, Germania) per il conteggio dei singoli fotoni correlati nel tempo (TCSPC) venivano utilizzate per l'acquisizione delle 7 curve di trasmittanza risolte nel tempo. L'immagine veniva acquisita mediante una scansione (raster scan) continua, registrando i dati ogni millimetro. Una scansione completa di una vista richiedeva in genere qualche minuto.

Successivamente, per aumentare il livello del segnale raccolto soprattutto alle lunghezze d'onda elevate, è stata rinnovata la catena di acquisizione, sostituendo il fascio di fibre di raccolta della luce e i fotomoltiplicatori con una sonda costituita da 8 fotomoltiplicatori a Silicio (SiPM, S13360-1350PE, Hamamatsu Photonics, Giappone) e le schede di acquisizione con un "time-to-digital converter" (TDC, SC-TDC1000/08 S, Surface Concept, Germania) a 8 canali.

Una descrizione dettagliata delle due configurazioni dello strumento è riportata in [3] e in [4], rispettivamente. 


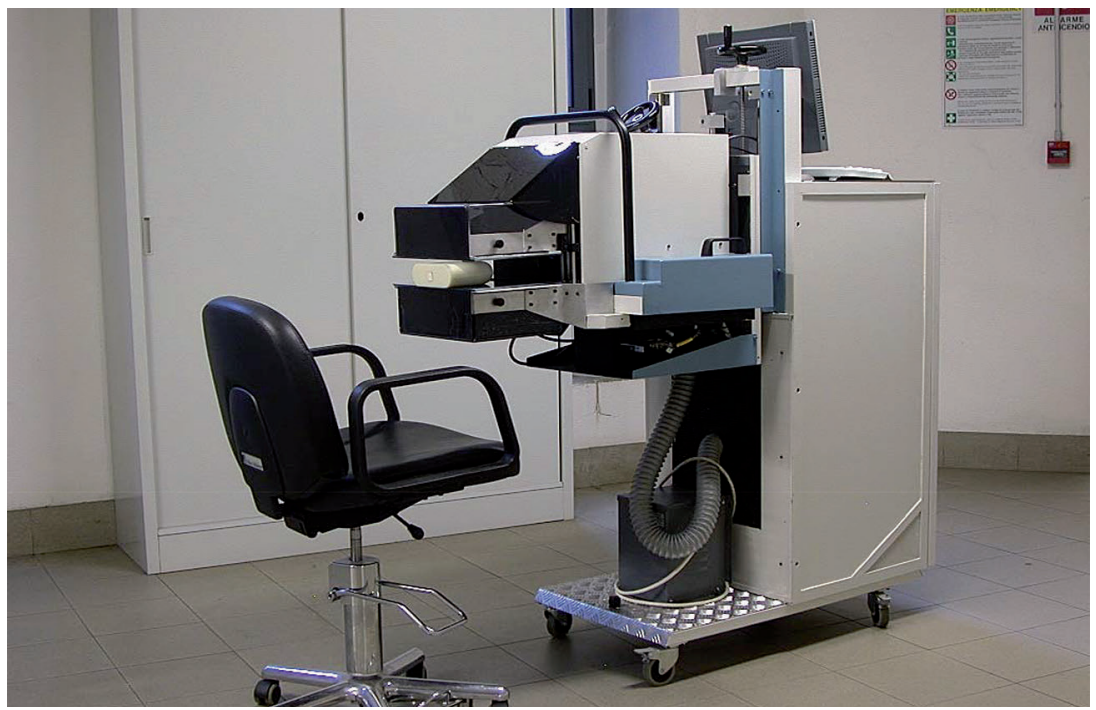

Fig. 1. Il mammografo ottico sviluppato dal Dipartimento di Fisica del Politecnico di Milano

\section{ANALISI DEI DATI}

\subsection{Modello omogeneo per la caratterizzazione media del tessuto mammario}

Le informazioni sulla composizione e la struttura del tessuto sono ottenute direttamente dalle curve risolte nel tempo misurate a 7 lunghezze d'onda. Viene applicata una procedura di interpolazione globale vincolata spettralmente, basata su una soluzione analitica dell'approssimazione di diffusione per una lastra omogenea infinita [5]. I parametri liberi sono le concentrazioni di ossi- e deossi-emoglobina $\left(\mathrm{HbO}_{2}\right.$ e $\mathrm{Hb}$, rispettivamente), acqua, lipidi e collagene, insieme ai parametri di scattering (ampiezza $a$ e potenza $b$ ).

La legge di Beer viene utilizzata per stimare il coefficiente di assorbimento a ciascuna lunghezza d'onda dalle concentrazioni dei principali costituenti del tessuto.

Conoscendo i parametri $a$ e $b$, il coefficiente di scattering ridotto viene modellato e ottenuto mediante una semplice approssimazione alla teoria di Mie: $\mu_{s}^{\prime}=a\left(\lambda / \lambda_{o}\right)^{-b}$, dove poniamo $\lambda_{o}=600 \mathrm{~nm}$ ed $a$ è il coefficiente di scattering interpolato $\mu_{s}^{\prime}\left(\lambda_{o}\right)[6]$. 


\subsection{Modello perturbativo per la caratterizzazione delle lesioni}

Al fine di caratterizzare otticamente le lesioni mammarie, assumiamo che possano essere trattate come perturbazioni di assorbimento localizzate, presenti in un mezzo diffusivo altrimenti omogeneo. Le curve di trasmittanza risolte nel tempo imperturbate e perturbate, rispettivamente $T_{0}(t)$ e $T(t)$, possono essere collegate sfruttando la legge di Lambert-Beer modificata:

$T(t)=T_{0}(t) e^{-\Delta \mu_{a} l(t)}$

dove $\lambda(t)$ è la lunghezza del percorso media percorsa nella disomogeneità dai fotoni rilevati al tempo $t$, mentre $\Delta \mu_{a}$ rappresenta la variazione di assorbimento all'interno della disomogeneità rispetto all'assorbimento omogeneo imperturbato $\mu_{a, 0}$. La lunghezza del percorso $l(t)$ può essere derivata come [7]:

$$
l(t)=-\frac{1}{T_{0}(t)} \frac{\partial T_{0}(t)}{\partial \mu_{a}}
$$

La curva imperturbata è ottenuta come media su una regione che esclude i bordi e le disomogeneità marcate. La curva perturbata sperimentale $T_{\text {pert }}$ è ottenuta da un'area ("area della lesione") centrata sulla corrispondente posizione della disomogeneità (cioè della lesione). L'area della lesione dipende dalla dimensione della lesione stessa. Per diametri di lesione $>15 \mathrm{~mm}$, è stata selezionata un'area di lesione di $9 \times$ $9 \mathrm{~mm}^{2}$, altrimenti $5 \times 5 \mathrm{~mm}^{2}$.

Partendo da $\Delta \mu_{a}$ e conoscendo il coefficiente di estinzione dei principali costituenti del tessuto mammario, dalla legge di Beer si stima la variazione delle concentrazioni $\Delta C_{i}$ tra lesione e tessuto sano (in termini di sangue, acqua, lipidi e collagene).

Questo metodo perturbativo si basa sulla conoscenza a priori del volume e della posizione della disomogeneità. Si è sempre ipotizzata una disomogeneità sferica posta a metà cammino tra sorgente e rivelatore. Per quanto riguarda le dimensioni si è considerata una sfera di volume equivalente in base al diametro massimo della lesione ottenuto dall'istopatologia, quando disponibile, o dalla mammografia o ecografia. 
I dettagli dell'implementazione del metodo perturbativo sono riportati in [3].

\section{RisUlTATI E DISCUSSIONE}

\subsection{Misura non invasiva della densità mammaria come fattore di rischio}

I principali costituenti del tessuto mammario (cioè acqua, lipidi, proteine strutturali e sangue) mostrano caratteristiche di assorbimento marcate e specifiche nell'intervallo spettrale NIR, come mostrato in Fig. 2. Il loro contributo all'assorbimento complessivo del tessuto varia in modo significativo con il tipo di mammella: passando da mammelle estremamente dense (cioè fortemente fibrose) ad adipose, l'assorbimento dell'acqua e del collagene può diminuire di un fattore 2 o anche 3 , mentre i lipidi aumentano corrispondentemente. Nel primo caso lo spettro è quindi dominato dall'assorbimento dell'acqua e si possono raggiungere valori molto elevati $\left(\mu_{a} \sim 0,35 \mathrm{~cm}^{-1}\right)$ sul picco dell'acqua a $975 \mathrm{~nm}$, come mostrato in Fig. 3a. Al contrario, l'assorbimento dei lipidi a $930 \mathrm{~nm}$ è la caratteristica principale nello spettro dei seni adiposi, ma l'assorbimento complessivo rimane relativamente basso a qualsiasi lunghezza d'onda (raggiungendo valori di circa $0,15 \mathrm{~cm}^{-1}$ a $\left.930 \mathrm{~nm}\right)$ [8].

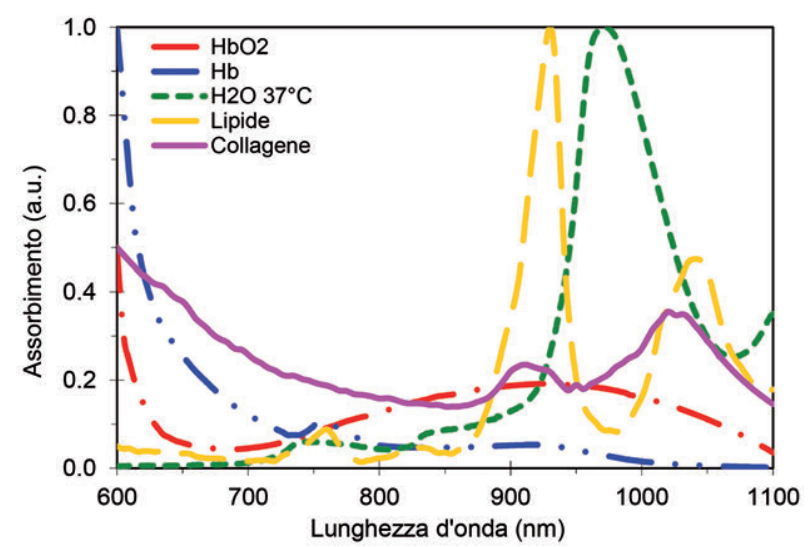

Fig. 2. Spettri di assorbimento normalizzati dei principali assorbitori di tessuto nel vicino infrarosso: deossi- e ossi-emoglobina ( $\mathrm{Hb}$ e $\mathrm{HbO}_{2}$, rispettivamente), acqua, lipidi e collagene. 
Il coefficiente di scattering ridotto diminuisce progressivamente all'aumentare della lunghezza d'onda, senza alcuna particolare caratteristica spettrale. Tuttavia, diversi tipi di seno hanno proprietà di scattering distinte, legate in particolare alla diversa pendenza dello spettro, con le mammelle fibrose caratterizzate da pendenze più ripide di quelle adipose (Fig. 3b). Questo, nell'approssimazione di Mie utilizzata nell'analisi dei dati, corrisponde ad un più elevato valore del parametro $b$.
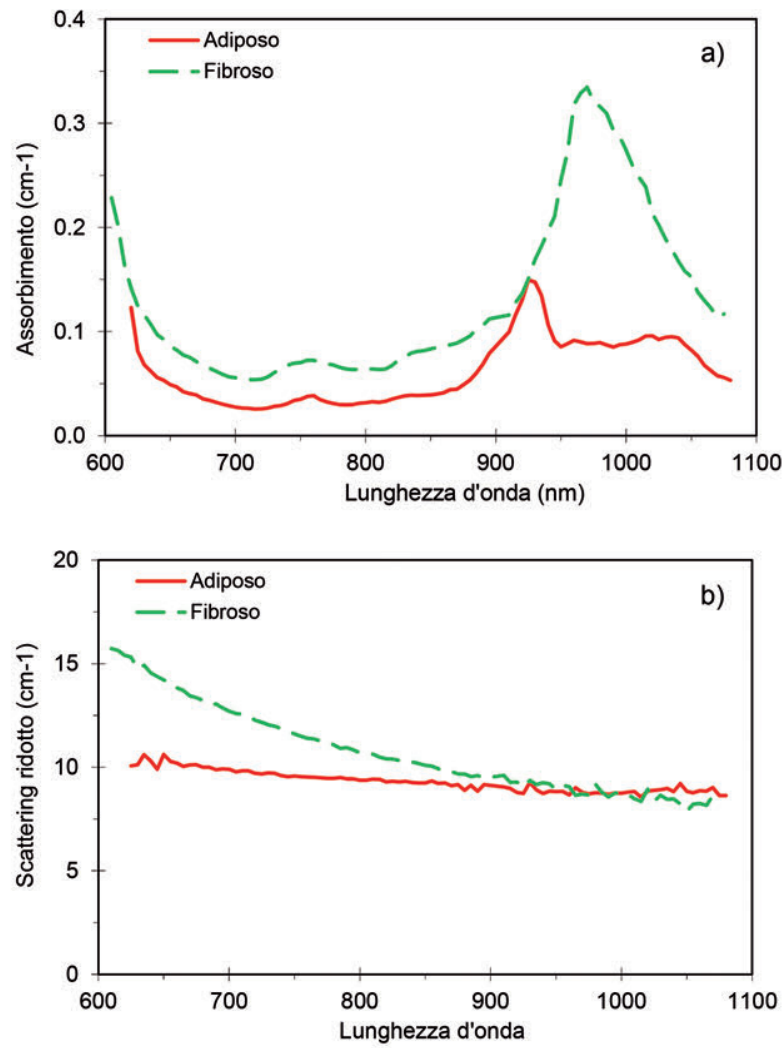

Fig. 3. Spettri tipici di assorbimento (a) e scattering ridotto (b) misurati in vivo da mammelle di diverso tipo: adiposo (solido) e fibroso (tratteggiato).

Più in generale, come decritto precedentemente, le proprietà ottiche stimate possono essere correlate alla composizione del tessuto (interpretando l'assorbimento con la legge di Beer) e alla struttura 
microscopica (descrivendo lo scattering con l'approssimazione empirica della teoria di Mie).

In questo modo è stata ottenuta una buona correlazione tra la composizione del tessuto derivata dalle misure ottiche e la densità della mammella. La densità è la frazione di tessuto fibroghiandolare ("denso", perché attenua i raggi $\mathrm{X}$ ) presente nel tessuto mammario, altrimenti sostanzialmente costituito da tessuto adiposo. A parte l'età, la densità è il più importante fattore di rischio indipendente per lo sviluppo del tumore della mammella. Attualmente viene derivata dall'analisi visuale delle immagini $X$. Il corrispondente fattore di rischio diventa quindi noto solo al momento della prima mammografia, tipicamente a 50 anni. Sarebbe invece molto utile avere a disposizione un metodo non invasivo, che non utilizzi radiazioni ionizzanti, per valutare la densità molto prima, dato che esistono interventi preventivi per ridurre la densità e il rischio correlato, non solo mediante l'assunzione di farmaci specifici, ma anche agendo sullo stile di vita (ad esempio, sul tipo di alimentazione).

In questa direzione l'ottica diffusa sta fornendo risultati molto promettenti. In particolare, in uno studio su più di 200 soggetti, in collaborazione con l'Istituto Europeo di Oncologia, lo strumento sviluppato dal Politecnico di Milano ha consentito di ottenere una buona correlazione $[9,10]$ tra la concentrazione di collagene e le classi BIRADS (Breast Imaging Reporting and Data System [11]), utilizzate nella pratica clinica per classificare la densità (da BIRADS 1 a BIRADS 4, per densità crescenti). Questo risultato rispecchia la fisiologia dei tessuti, dato che un'elevata densità indica un'elevata frazione fibroghiandolare, più ricca di collagene rispetto al tessuto adiposo. In accordo con questa osservazione è anche la buona correlazione ottenuta tra la pendenza $b$ dello spettro di scattering e le classi BIRADS. Il tessuto ricco di collagene è infatti caratterizzato da uno spettro si scattering molto pendente, probabilmente a causa della struttura microscopica del collagene nel tessuto.

\section{DiSCRIMINAZIONE TRA LESIONI MALIGNE E BENIGNE}

L'utilizzo di lunghezze d'onda elevate (rosso e NIR) garantisce non invasività all'ottica diffusa. Allo stesso tempo, però, essendo molto probabili gli eventi di scattering, i fotoni non procedono secondo traiet- 
torie rettilinee e questo limita significativamente la risoluzione spaziale della tecnica. La risoluzione dipende dalle dimensioni della disomogeneità ottica che si deve rivelare, dalla sua profondità e dal suo contrasto ottico, ma difficilmente può essere migliore di alcuni millimetri e spesso, in condizioni reali di misura, è inferiore [12]. L'ottica diffusa non è quindi adatta alla diagnosi basata sulle caratteristiche morfologiche. La capacità di stimare la composizione del tessuto, insieme a importanti parametri sanguigni (volume totale di emoglobina $\mathrm{HbT}$ e livello di ossigenazione $\mathrm{SO}_{2}$ ) possono però essere di aiuto nella discriminazione tra lesioni maligne e benigne. Lo stesso studio già citato nella sezione precedente ha anche consentito di confrontare la composizione di lesioni maligne e benigne, derivata otticamente.

Tutti i tipi di lesioni analizzate avevano mediamente un maggiore contenuto di emoglobina e di collagene rispetto al tessuto sano nella stessa mammella, ma la differenza è risultata più marcata per le lesioni maligne [13]. Un algoritmo di "machine learning" per il "boosting" adattativo è stato poi applicato utilizzando come variabili per la discriminazione la composizione del tessuto ottenuta dalle misure ottiche, insieme ad informazioni note dall'anamnesi delle pazienti e che rappresentano fattori di rischio (ad esempio, età, numero di gravidanze, terapia ormonale sostitutiva, ecc.). In questo modo le lesioni maligne sono state discriminate da quelle benigne con sensibilità $=87.7 \pm 1.98 \%$, and specificità $=78.7 \pm 3.0 \%[14]$.

Il risultato è abbastanza promettente, anche se si deve tenere conto del fatto che è stato ottenuto da un limitato numero di casi $(N=84)$.

\section{Approccio multimodale alla DiagnOSI DEL TUMORE DELLA MAMMELLA: IL PROGETTO SOLUS}

L'ecografia (ultrasuoni - US) è la tecnica di prima scelta per valutare le lesioni non palpabili visibili alla mammografia e le lesioni palpabili e anche per guidare la biopsia mammaria. Le caratteristiche morfologiche sono state classificate per distinguere tra lesioni maligne e benigne. Attualmente, però, i risultati diagnostici degli US convenzionali (in modalità B) non sono del tutto soddisfacenti e per una diagnosi definitiva si ricorre alla biopsia, che in molti casi si dimostra poi inutile (falso positivo).

Recentemente, è stata introdotta una tecnica a ultrasuoni più avanzata, detta elastografia (shear wave elastography - SWE). 
L'elastografia fornisce una misura quantitativa e riproducibile della rigidezza dei tessuti e sfrutta la maggiore presenza di matrice extracellulare nel tessuto neoplastico come marker di malignità. Una recente metaanalisi ha valutato l'efficacia dell'elastografia per la diagnosi del tumore della mammella: la specificità degli US convenzionali è risultata del $55 \%$, mentre l'aggiunta dell'elastografia aumenta la specificità all' $80 \%$, lasciando la sensibilità sostanzialmente inalterata al 95\% [15]. Tuttavia, è necessario un ulteriore miglioramento della specificità per ottenere una riduzione significativa del tasso di falsi positivi e generare un impatto reale sia sulle pazienti (evitando un inutile esame invasivo) che sui sistemi sanitari (riducendo la spesa per le biopsie).

Il progetto SOLUS - "Smart Optical and Ultrasound Diagnostics of Breast Cancer" [16], finanziato nell'ambito del programma quadro europeo H2020, ha proprio lo scopo di sviluppare un sistema di imaging multimodale per migliorare in modo non invasivo la specificità della diagnosi del tumore della mammella, discriminando in particolare le lesioni che sono al limite tra benigne e maligne.

Lo sviluppo della sonda multimodale SOLUS è partito da uno strumento per imaging a US (Aixplorer ${ }^{\circledR}$ Mach30, commercializzato da Supersonic Imagine, partner del progetto), che consente di acquisire immagini in modalità $\mathrm{B}$ ed effettuare la misura della rigidezza mediante SWE. Per poter valutare la composizione del tessuto nello stesso volume analizzato dagli US, attorno al trasduttore US sono stati aggiunti otto punti di misura ottica che consentono di effettuare una tomografia ottica diffusa a 8 lunghezze d'onda nel dominio del tempo. Per ottenere questo risultato, è stato necessario combinare elevate performance e piccole dimensioni. Sono stati quindi sviluppati tutti gli elementi del sistema: laser impulsati a picosecondi a 8 lunghezze d'onda (630-1060 $\mathrm{nm}$ ), rivelatore SiPM di area elevata (per ottenere elevata sensibilità) con gate temporale (per poter gestire segnali che variano su vari ordini di grandezza al variare della distanza tra sorgente e rivelatore) ed elettronica di acquisizione di segnali nel dominio del tempo. L'insieme di tutti questi elementi è racchiuso in un volume con sezione di circa $1 \mathrm{~cm}^{2}$ e lunghezza di circa $4 \mathrm{~cm}$, ordini di grandezza più piccolo del volume occupato dagli strumenti finora disponibili per eseguire lo stesso tipo di misure con performance analoghe.

La sonda è attualmente in fase di caratterizzazione. Appena questa sarà conclusa, inizierà la valutazione clinica del sistema SOLUS per la discriminazione tra lesioni maligne e benigne. 


\section{BIBLIOGRAFIA}

1. Contini D, Martelli F, Zaccanti G, Photon migration through a turbid slab described by a model based on diffusion approximation. I. Theory, Appl Opt., 1997: 36:4587-4599.

2. Fishkin JB, Gratton E, Propagation of photon-density waves in strongly scattering media containing an absorbing semi-infinite plane bounded by a straight edge. $J$ Opt Soc Am A., 1993: 10: 127.

3. Taroni P, Pifferi A, Salvagnini E, Spinelli L, Torricelli A, Cubeddu R, Seven-wavelength time-resolved optical mammography extending beyond $1000 \mathrm{~nm}$ for breast collagen quantification, Opt Express, 2009: 17(18):15932-15946.

4. Ferocino E, Martinenghi E, Mora AD, Pifferi A, Cubeddu R, Taroni P, High throughput detection chain for time domain optical mammography, Biomed Opt Express., 2018: 9: 755-770.

5. D’Andrea C, Spinelli L, Bassi A, Giusto A, Contini D, Swartling J, Torricelli A, Cubeddu R, Time-resolved spectrally constrained method for the quantification of chromophore concentrations and scattering parameters in diffusing media, Opt. Express, 2006. 14: 1888-1898.

6. Nguyen J, Hayakawa CK, Mourant JR, Spanier J, Perturbation Monte Carlo methods for tissue structure alterations, Biomed Opt Express, 2013: 4: 1946-1963.

7. Del Bianco S, Martelli F, Zaccanti G, Penetration depth of light re-emitted by a diffusive medium: theoretical and experimental investigation, Phys Med Biol, 2002: 47: 4131-4144.

8. Taroni P, Quarto G, Pifferi A, Abbate F, Balestreri N, Menna S, Cassano E, Cubeddu R, Breast tissue composition and its dependence on demographic risk factors for breast cancer: Non-invasive assessment by Time Domain diffuse optical spectroscopy, PLoS One, 2015: 10: e0128941.

9. Taroni P, Quarto G, Pifferi A, Ieva F, Paganoni AM, Abbate F, Balestreri N, Cassano E, Cubeddu R, Optical identification of subjects at high risk for developing breast cancer, J Biomed Opt, 2013: 18: 060507.

10. Taroni P, Pifferi A, Quarto G, Spinelli L, Torricelli A, Abbate F, Balestreri N, Cassano E, Cubeddu R, Noninvasive assessment of breast cancer risk using timeresolved diffuse optical spectroscopy. J Biomed Opt, 2010: 15: 060501.

11. BI-RADS® - Mammography 2013 - American College of Radiology.

12. Cubeddu R, Pifferi A, Taroni P, Torricelli A, Valentini G, Spatial resolution of imaging with diffusing light: Edge spread function measurements on a realistic tissue phantom, Med Phys, 1999: 26: 462-471.

13. Quarto G, Spinelli L, Pifferi A, Torricelli A, Cubeddu R, Abbate F, Balestreri N, Menna S, Cassano E, Taroni P, Estimate of tissue composition in malignant and benign breast lesions by time-domain optical mammography. Biomed Opt Express, 2014: 5: 3684-3698.

14. Taroni P, Paganoni AM, Ieva F, Pifferi A, Quarto G, Abbate F, et al. Non-invasive optical estimate of tissue composition to differentiate malignant from benign breast lesions: A pilot study. Sci Rep, 2017: 7: 40683.

15. Liu B, Zheng Y, Huang G, Lin M, Shan Q, Lu Y, Tian W, Xie X, Breast Lesions: 
Quantitative Diagnosis Using Ultrasound Shear Wave Elastography-A Systematic Review and Meta-Analysis. Ultrasound Med Biol, 2016: 42: 835-847.

16. SOLUS - Smart Optical and Ultrasound Diagnostics of Breast Cancer [Internet]. Progetto H2020 No. 731877. http://solus-project.eu/ 
